\title{
Pulmonary metastasis from gastric cancer: A case report and review of literature
}

\author{
Motohisa Kuwahara ${ }^{1^{\star}}$, Mari Matsumoto ${ }^{1}$, Kazuya Naritomi $^{1}$, Masae Mano $^{1}$, Daisuke Hamatake ${ }^{2}$, \\ Koji Inutsuka², Takayuki Shirakusa ${ }^{2}$, Akinori Iwasaki ${ }^{2}$
}

${ }^{1}$ Division of Surgery, Saiseikai Futukaichi Hospital, Fukuoka, Japan; ${ }^{*}$ Corresponding Author: KHC03152@,nifty.com

${ }^{2}$ Departement of Chest Surgery, Fukuoka University Hospital, Fukuoka, Japan

Received 7 July 2013; revised 5 August 2013; accepted 12 August 2013

Copyright (C) 2013 Motohisa Kuwahara et al. This is an open access article distributed under the Creative Commons Attribution License, which permits unrestricted use, distribution, and reproduction in any medium, provided the original work is properly cited.

\begin{abstract}
We present a case report of a 63-year-old male who underwent lung resections for metastases originating from gastric cancer 18-year after total gastrectomy with lymphadenectomy. The gastrectomy was performed in 1994; histological examination of the original tumor revealed stage II poorly differentiated adenocarcinoma [pT2 (MP), N0, M0]. Chest X-ray and computed tomography in 2012 showed a well-defined tumor, 9 $\mathrm{mm}$ in size, at the left $\mathrm{S} 3$ of the lung. Thoracoscopic partial resection was performed. The tumor was diagnosed as poorly differentiated carcinoma, most likely metastatic gastric adenocarcinoma. Although rarely performed, resection of pulmonary metastases from carcinoma of the stomach was done to improve the patient's chances for long-term survival.
\end{abstract}

Keywords: Gastric Cancer; Metastatic Lung Cancer; Metastasectomy

\section{INTRODUCTION}

Gastric cancers are associated with a higher incidence of liver metastasis, which has a low radical resectability rate. Even without disseminated or multiple liver metastasis, pulmonary metastasis typically has carcinomatous lymphangitis or carcinomatous pleuritis. In contrast, nodular lesions are rare. Surgical resection is therefore usually not indicated. We herein describe a patient with proven pulmonary metastasis of gastric carcinoma 18year-after total gastrectomy.

\section{CLINICAL SUMMARY}

A 45-year-old male underwent a total gastrectomy for gastric cancer in July 1994 (Figure 1(A)). He had no history of drinking or smoking, and his past medical and family histories were unremarkable. Histological examination revealed a poorly differentiated adenocarcinoma, which was classified as pT2 (MP) N0M0, stage II. Blood chemistry analyses were unremarkable, and tumor markers (i.e., carcinoembryonic antigen and carbohydrate antigen 19 - 9) were all within normal limits. Following gastrectomy, the patient was asymptomatic and continued follow-up for 5 years at an out-patient clinic. Then he underwent a medical examination yearly.

In November 2011 (63 years old), chest X-ray revealed an approximately $1-\mathrm{cm}$ nodular shadow in the left upper lung field. Chest computed tomography (CT) revealed a tumor $9 \times 8 \mathrm{~mm}$ in size, at the subpleural portion in left S3 of the lung (Figure 1(B)). The well-defined tumor had a CT value of 60 Hounsfield Units (HU) and enhanced CT values of $210 \mathrm{HU}$ after 40 seconds and $100 \mathrm{HU}$ after 2 minutes. Fluorodexyglucose accumulation (maximum standardized uptake value $\left[\mathrm{SUV}_{\max }\right]=$ 2.8) of was shown on fluorodexyglucose positron emission tomography (FDG-PET) (Figure 1(C)). Fiberscopic evaluation of the upper intestine and total colon found no abnormalities. Initially, we thought that the lung tumor was either primary or metastatic lung cancer. Surgery was indicated, even for pulmonary metastases, because there was no evidence of recurrence at extrapulmonary sites, such as the primary site or the liver, by FDG-PET.

The tumors in the left lung were resected thoracoscopically, and were well defined and red in color (Figure 1(D)).

Histologically, the tumor consisted of solidly grown eosinophilic polygonal cells (Figure 2(A)), and had the following immunostaining properties: cytokeratin (CK) 7-negative, TTF-1-negative and CK 20-weakly positive (Figures 2(B) and (C)). The tumor was diagnosed as poorly differentiated carcinoma, most likely metastatic adenocarcinoma, accorded with the previous gastric cancer from the pathological report.

The patient was discharged 10 days after partial resection without any unusual events. After pulmonary resec- 

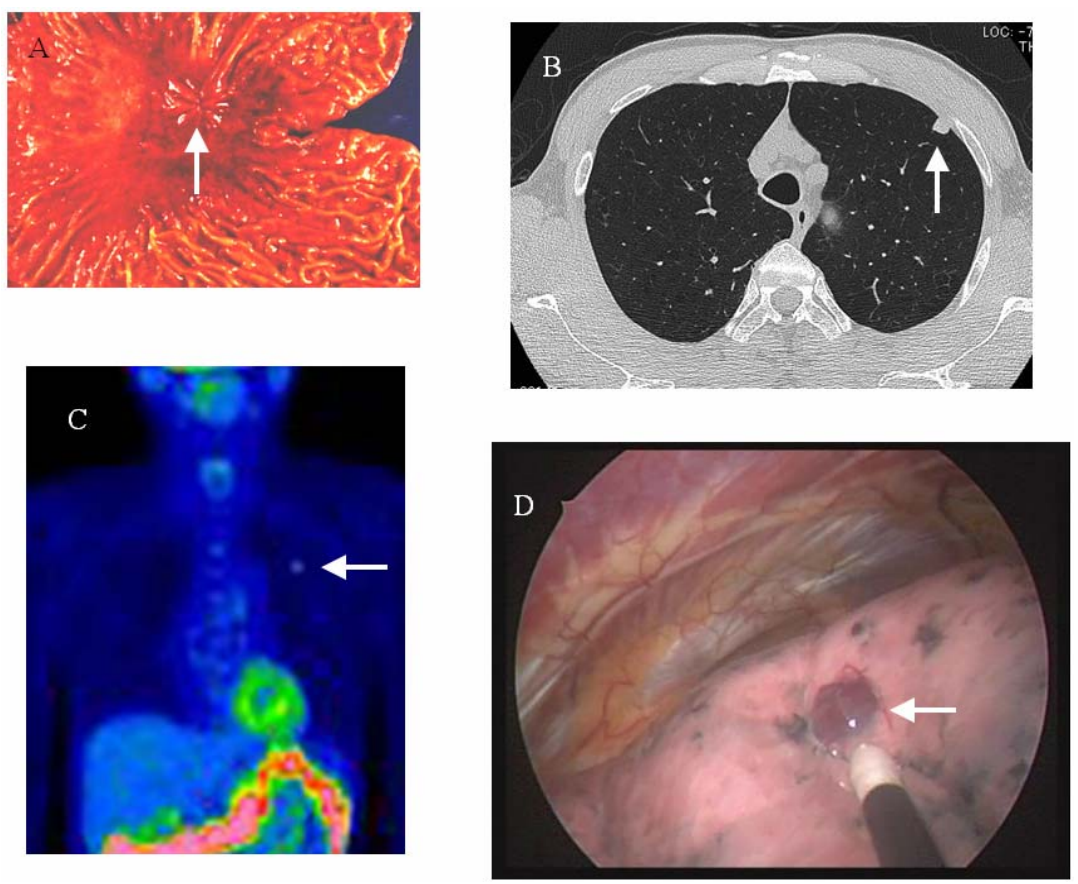

Figure 1. (A) Resected specimen of gastric cancer. (B) Chest CT showed a tumor 9 $\times 8 \mathrm{~mm}$ in size at the subpleural portion in the left $\mathrm{S} 3$ of the lung. The well-defined tumor had a CT value of 60 Hounsfield Units. (C) FDG accumulation was shown by PET $\left(\mathrm{SUV}_{\max }=2.8\right)$. (D) Thoracoscopic findings showed that the tumor was well defined and red in color.
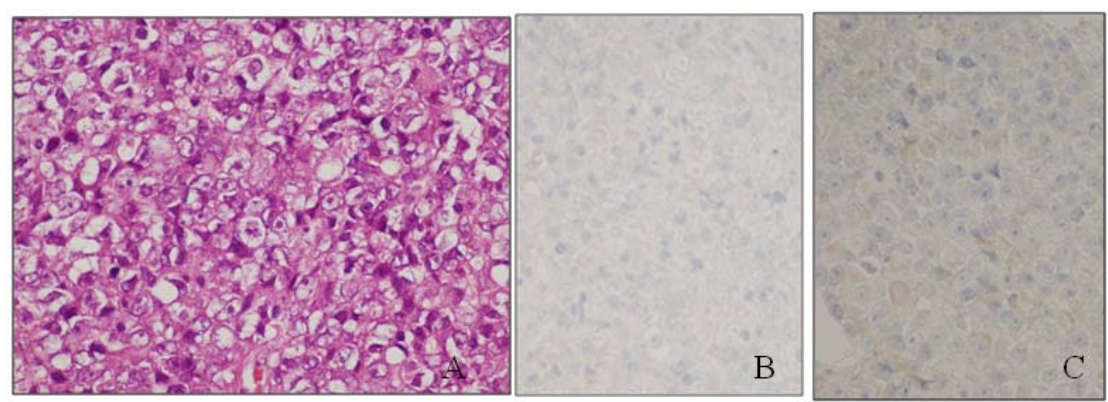

Figure 2. (A) Histological findings of poorly differentiated carcinoma of the left lung (hematoxylin and eosin staining). (B) Immunohistological findings of TTF-1 immunostaining. (C) Immunohistological findings of CK 20 immunostaining.

tion, we provided follow-up care at our out-patient clinic for 15 months. There were not new nodules on chest CT, and fiberscopic evaluation found no abnormalities in the upper intestine or total colon. Based on the clinical course and pathological-futures, we concluded that the patient had pulmonary metastasis of gastric carcinoma 18 years after total gastrectomy for gastric cancer.

\section{DISCUSSION}

Based on autopsy results, the incidence of pulmonary metastatic lung cancer from gastric cancer has been reported at $49.6 \%$ [1]. Surgical resection is rarely indicated for recurrent or metastatic gastric cancer because it tends to occur at multiple sites and shows aggressive biological behaviors [2]. It is reasonable to assume that the lung metastases in these case were from the stomach because there were multiple, small lung lesions, unlike the single location of their gastric neoplasm. In our case, the tumor was well defined and round and there was a subpleural portion. The tumor was not stained by TTF-1 for pulmonary tumor maker. We therefore thought that the pulmonary tumor was a metastatic lung cancer.

The patient was asymptomatic and had no recognizable neoplasm by gastrointestinal fiberscope findings, chest and abdominal CT, or FDG-PET findings between the perioperative period and 15 month follow-up. No new lesions have arisen at any pulmonary or another sites. 
Table 1. Characteristics of cases of pulmonary metastasectomy for gastric cancer.

\begin{tabular}{|c|c|c|c|c|c|c|c|c|c|}
\hline Author & Year & Age & Sex & Histology & Stomach & pTNM & DFI & $\begin{array}{l}\text { Type of } \\
\text { Resection }\end{array}$ & Outcome \\
\hline \multirow[t]{4}{*}{ Kanemitsu ${ }^{4)}$} & 1998 & 63 & Male & Differentiated & Total & $\mathrm{T} 2 \mathrm{~N} 2 \mathrm{M} 0$ & 22 & Lobectomy & Dead; 23 \\
\hline & & 53 & Male & Differentiated & Total & T3N2M0 & 39 & Lobectomy & Dead; 45 \\
\hline & & 66 & Male & Differentiated & Total & $\mathrm{T} 3 \mathrm{~N} 2 \mathrm{M} 0$ & 48 & Lobectomy & Dead; 10 \\
\hline & & 70 & Male & Differentiated & Total & $\mathrm{T} 2 \mathrm{~N} 2 \mathrm{M} 0$ & 19 & Lobectomy & Dead; 19 \\
\hline Nakahashi $^{5)}$ & 2004 & 61 & Male & Moderately differ. & Total & T3N1M0 & 24 & RML & Alive; 84 \\
\hline \multirow[t]{2}{*}{ Nakayama $^{6}$} & 2008 & 62 & Male & Moderately differ. & Distal & $\mathrm{T} 2 \mathrm{~N} 1 \mathrm{M} 0$ & 38 & RML & \\
\hline & & & & & & & 48 & LLL & Alive; 65 \\
\hline Tanai $^{7}$ & 2010 & 61 & Male & Well differ. & Distal & T3N1M0 & 17 & Wedge & Alive; 65 \\
\hline Hanyu ${ }^{8)}$ & 2010 & 51 & Female & Moderately differ. & Total & T4N2M0 & 85 & RML & Alive; 19 \\
\hline Ours & 2012 & 63 & Male & Poorly differ. & Total & T2N0M0 & 212 & Wedge & Alive; 18 \\
\hline
\end{tabular}

Abbreviations: DFI: disease free interval differ: differentiated carcinoma, RML: right middle lobe lobectomy, LLL: left lower lobe lobectomy.

The tumors were weakly positive for CK 20, a gastrointestinal maker. A combination of $\mathrm{TTF}-1^{-} \mathrm{CK} 20^{+}$was highly significantly associated with adenocarcinoma of gastrointestinal origin [3]. The sensitivity and specificity of this combination of immunomarkers for a metastatic adenocarcinoma from gastrointestinal tract were $79 \%$ and $100 \%$. He had past history of resected gastric cancer. The tumor accorded with the previous gastric cancer from the pathological report, was diagnosed as poorly differentiated carcinoma, most likely metastatic gastric adenocarcinoma.

Surgical resectable solitary metastasis only occurs in $0.1 \%$ in patients who have undergone curative resection for gastric cancer [4]. By the end of 20th century, the time interval to recurrence following pulmonary resection ranged from 6 to 36 months, all the median survival after recurrence was 24.3 months. Several cases of resection of solitary pulmonary metastasis from gastric cancer have been reported (Table 1) [4-8]. In recent years, advances in anesthesia and thoracotomy procedure have improved survival, and improvements in high-resolution CT and FDG-PET have increased detection. Carefully selected patients have good chance of successful pulmonary metastasectomy for gastric cancer.

\section{CONCLUSION}

In summary, we describe a case of pulmonary metastases from gastric carcinoma that was treated with surgical resection. At least empirically, surgery may be considered a treatment of choice to improve the long-term survival in such patients.

\section{REFERENCES}

[1] Ishii, T., Ikegami, N., Hosoda, Y., Koide, O. and Kaneko, M. (1981) The biological behavior of gastric cancer. The
Journal of Pathology, 134, 97-115. doi:10.1002/path.1711340202

[2] Lee, H., Chen, F., Lo, C., Wang, C., Lo, W. and Luh, S. (2010) Metastasis of gastric carcinoma to the thyroid and lung: A case report and review of literature. Journal of Zhejiang University-Science B, 11, 542-546. doi:10.1631/jzus.B0900378

[3] Su, Y., Hsu, Y. and Chai, C. (2006) Role of TTF-1, CK20, and CK7 immunohistochemistry for diagnosis of primary and secondary lung adenocarcinoma. Kaohsiung Journal of Medical Sciences, 22, 14-19. doi:10.1016/S1607-551X(09)70214-1

[4] Kanemitsu, Y., Kondo, H., Katai, H., et al. (1998) Surgical resection of pulmonary metastases from gastric cancer. Journal of Surgical Oncology, 69, 147-159.

doi:10.1002/(SICI)1096-9098(199811)69:3<147::AID-JS O5>3.0.CO;2-H

[5] Nakahashi, C., Kinoshita, T., Konishi, M., et al. (2004) Long-term survival achieved by repeated resection of metachronous pulmonary and adrenal metastases of afetoprotein-producing gastric cancer: Report of a case. Surgery Today, 34, 784-787. doi:10.1007/s00595-004-2796-3

[6] Nakayama, H., Ichinose, S., Kato, Y., Ito, H., Masui, K. and Kameda, Y. (2008) Long-term survival after a surgical resection of pulmonary metastases from gastric cancer: Report of a case. Surgery Today, 38, 150-153. doi:10.1007/s00595-007-3577-6

[7] Tanai, C., Hamaguchi, T., Watanabe, S., Katai, H., Tochigi, N. and Shimada, Y. (2010) A case of long-term survival after surgical resection of solitary pulmonary metastasis from gastric cancer. Japanese Journal of Clinical Oncology, 40, 85-89. doi:10.1093/ijco/hyp098

[8] Hanyu, T., Kanda, T., Matsuki, A., et al. (2010) Endobronchial metastasis from adenocarcinoma of gastric cardia 7 years after potentially curable resection. World Journal of Gastrointestinal Surgery, 2, 270-274. doi: $10.4240 /$ wigs.v2.i8.270 